\title{
The Impact of Antibiotic-Loaded Bone Cement on Antibiotic Resistance in Periprosthetic Knee Infections
}

\author{
Daniel R. Schmitt, MD, Cameron Killen, MD, Michael Murphy, MS, Michael Perry, MD, \\ Joseph Romano, MD, Nicholas Brown, MD \\ Department of Orthopaedic Surgery, Loyola University Medical Center, Maywood, IL, USA
}

Background: Antibiotic-loaded bone cement (ALBC) is commonly used in total knee arthroplasty (TKA), especially among high-risk patients. While previous studies have reported on the efficacy of ALBC in reducing the rate of periprosthetic joint infection (PJI), its impact on antibiotic resistance has not been determined. The purpose of this study was to investigate antibiotic resistance among organisms causing PJIs after TKA in which ALBC was utilized.

Methods: A retrospective review from December 1998 through December 2017 identified 36 PJIs that met inclusion criteria. Patients with culture-negative infection and unknown cement type were excluded. Patient characteristics, infecting organism, and antibiotic susceptibilities were recorded. ABLC included an aminoglycoside in all cases.

Results: There was no difference in the type of PJI between the 2 groups. Staphylococcus species was the most commonly isolated, with 9 of 16 cases (56.3\%) using non-ALBC and 14 of 20 (65.0\%) cases using ALBC. Of those infected with Staphylococcus, there was no significant difference in antibiotic susceptibilities between groups. Overall, there were only 3 cases where the infecting organism was aminoglycoside resistant (standard cement, 1; ALBC, 2).

Conclusions: These results suggest that the use of ALBC does not increase the risk of antibiotic resistance or affect the pattern of infection, even as the use of ALBC continues to increase, particularly among high-risk patients.

Keywords: Infection, Total knee arthroplasty, Periprosthetic knee infections, Antibiotic resistance, Antibiotic laden bone cement

The rate of deep infection in primary total knee arthroplasty (TKA) has remained relatively steady at around $1 \%-2 \%$ in most published series. ${ }^{1)}$ It remains one of the most devastating and costly complications following joint replacement. Antibiotic-loaded bone cement (ALBC) has been used as a measure to prevent prosthesis-related infections since the 1970s. ${ }^{2)}$ Gentamicin or a similar aminoglycoside has been used frequently because of its water

Received April 19, 2019; Accepted January 8, 2020

Correspondence to: Daniel R. Schmitt, MD

Department of Orthopaedic Surgery, Loyola University Medical Center, 2160

S. First Ave. Maywood, IL 60153, USA

Tel: +1-708-216-9000, Fax: +1-708-226-9348

E-mail: dan.schmittmd@gmail.com solubility, chemical stability, low allergenicity, and thermal stability, in addition to its ability to theoretically confer long-term protection to the local tissues. ${ }^{3)}$ Currently, the use of antibiotics in bone cement has become commonplace in both single-stage and 2-stage revision TKA to reduce the rate of reinfection, particularly in higher risk populations. $^{4)}$

The use of ALBC in the setting of primary total joint arthroplasties has also become increasingly common as literature examining European registry data has shown a potential benefit of ALBC, particularly in total hip arthroplasty (THA). ${ }^{5-7)}$ While the evidence for the use of ALBC in primary TKA is both limited and conflicting, the use of ALBC among patients undergoing TKA has increased in the United States, particularly among high-risk 
Schmitt et al. Antibiotic Resistance Due to Antibiotic-Laden Bone Cement

Clinics in Orthopedic Surgery • Vol. 12, No. 3, $2020 \bullet$ www.ecios.org

patients. However, given the conflicting literature available on the efficacy of ALBC in TKA, ${ }^{8-15)}$ with the increasing focus on preventing superinfections and spawning new resistant organisms, there is a need to better determine whether routine use of ALBC in primary TKA is effective in decreasing deep infections without leading to antibiotic resistance.

A small number of prior studies have demonstrated data concerning the development of antibiotic resistance with the use of ALBC in hip and knee arthroplasties. Thomes el al. ${ }^{16)}$ demonstrated a higher rate of gentamicin-resistant infections among arthroplasties utilizing gentamicin-loaded bone cement compared with plain bone cement in a rat model. Additionally, Sanzen and Walder ${ }^{17)}$ and Hope et al. ${ }^{18)}$ have reported increased rates of gentamicin-resistent coagulase-negative Staphylococcus (CNS) associated with the use of gentamicin-loaded bone cement.

The purpose of this study was to investigate antibiotic resistance among organisms causing periprosthetic knee infections after TKA in which ALBC was utilized. The hypothesis was that there would be a higher incidence of aminoglycoside-resistant organisms in periprosthetic joint infections (PJIs) in patients whose primary TKA was performed with ALBC as compared to standard cement. The second hypothesis was that there would be a difference in the pattern of infecting organism between these groups.

\section{METHODS}

This study was a retrospective chart review. After obtaining the Institutional Review Board approval (IRB No. LU 210848), our institutional surgical procedure database was queried from January 1998 through December 2017 for all cases of periprosthetic knee infection.

Patients were excluded from the study if the cement type (standard cement versus ALBC) used in the initial primary TKA was unknown, if the infection was culture negative, or if the antibiotic susceptibilities were not available for the organism isolated during the infectious workup. Inclusion criteria were satisfied for 36 PJIs occurring in 32 unique patients. Of the 65 patients excluded, 7 were excluded due to culture-negative results or lack of antibiotic susceptibilities. The remaining 58 patients were excluded because either the index TKA was performed at an outside institution or the operative report either was not available or did not include the type of cement used in the index procedure. After excluding 65 patients based on these criteria, 36 periprosthetic knee infections in 32 unique pa- tients were included in the final cohort. There were 5 acute postoperative PJIs (within 4 weeks of index procedure), 21 acute hematogenous infections, and 10 chronic infections. Of these, 20 had ALBC and 16 had cement without antibiotics. Appropriate antibiotic prophylaxis based on the American Academy of Orthopaedic Surgeons (AAOS) guidelines was given in all cases. In patients who received antibiotic bone cement, pre-mixed cement from the manufacturer was utilized with $1 \mathrm{~g}$ of either gentamycin or tobramycin per $40 \mathrm{~g}$ bag of cement.

The procedures were performed by 5 surgeons over a 20-year period with no institutional protocol on when to use antibiotic cement. Some surgeons never used it, others had subjective personal criteria, and others used it in every case. Also, practice patterns changed over the study period. Therefore, this provided a unique opportunity to study the effect of antibiotic cement on organism resistance as the utilization of antibiotic cement was relatively unbiased and effectively randomized. To control for the potential difference in patient characteristics between the ALBC versus normal cement cohorts, Charlson Comorbidity Index and diabetes incidences were analyzed in the infected patients.

The chart review was performed using Loyola University Medical Center's electronic medical record (EPIC Hyperspace; EPIC Systems Inc., Verona, WI, USA) to identify patient demographics, which included body mass index, history of diabetes mellitus, tobacco, and other comorbid risk factors included in the Charlson Comorbidity Index. Operative reports were reviewed for cement type used. If cement type was not found within the operative report, the surgical encounter was reviewed for billing information as it pertained to the cement type used. Infecting organism and minimum inhibitory concentrations (MIC) of all antibiotics tested by the Loyola University Medical Center microbiology laboratory were also recorded.

The major outcomes were (1) the effect of ALBC on antibiotic resistance among infecting organisms based on MIC and (2) species of infecting organism in patients with PJI. Data were analyzed using Fisher exact and chi-square tests with significance for $p$-values $<0.05$. A power analysis was performed, which demonstrated that a minimum of 17 patients in each group would be needed to detect a difference in aminoglycoside resistance of $15 \%$ versus $60 \%$ with an alpha value of 0.05 and power of 0.80 .

\section{RESULTS}

Overall, 20 of the infections occurred in cases using ALBC while 16 infections occurred in cases using non-ALBC. 
Two of 20 patients (10\%) in the ALBC group versus 1 of 16 of patients $(6.3 \%)$ in the non-ALBC group showed aminoglycoside resistance $(p>0.99)$. Staphylococcus species was isolated in 9 of 16 cases using non-ALBC (56.3\%) and 14 of 20 cases using ALBC (70\%), making it the most commonly isolated organism ( $p=0.49)$ (Figs. 1 and 2). Of the 9 Staphylococcus infections in cases using non-ALBC, 6 were Staphylococcus aureus while the remaining 3 were CNS. Of the 14 Staphylococcus infections in cases using non-ALBC, 9 were $S$. aureus while the remaining 5 were CNS. Only 1 of 14 (7.1\%) of the Staphylococcus infections was aminoglycoside-resistant organisms in the ALBC group versus 1 of $8(12.5 \%)$ in the non-ALBC group $(p>$ 0.99 ). Additionally, there were no differences noted in susceptibilities (as determined by MIC) among the remaining 8 antibiotics tested for all infections per our institution's microbiology lab protocol for Staphylococcus infections (Table 1).

Streptococcus species was the second most commonly isolated organism in both the ALBC and standard bone cement groups, being isolated in 5 of 16 (31\%) and 4 of 20 (20\%), respectively $(p=0.47)$. Pseudomonas was isolated in 2 of 15 cases using standard bone cement (13\%), which was the only gram-negative organism isolated in either group. Enterococcus species was isolated in the remaining infections in the ALBC group (2/20,10\%).

There was no statistical difference in Charlson Comorbidity Index between the 2 groups (mean, 3.44 vs. 2.80 for ALBC and non-ALBC, respectively; $p=0.457$ ). The ALBC group had 6 of 20 patients as diabetic, while the non-ALBC group had 3 of 16 patients as diabetic, which was not statistically significantly different $(p=0.445)$.

\section{DISCUSSION}

The purpose of this study was to investigate the develop-

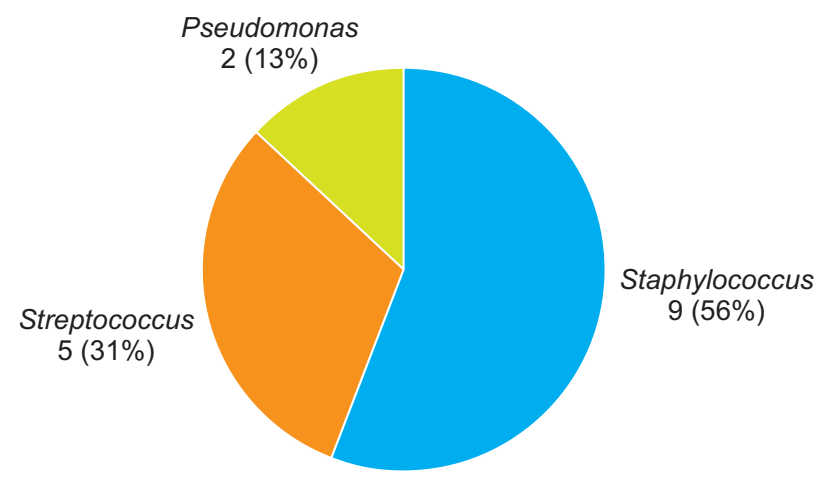

Fig. 1. Infecting organism in total knee arthroplasty utilizing standard bone cement. ment of antibiotic resistance among organisms causing periprosthetic knee infections in cases where ALBC was utilized during the index arthroplasty procedure. While there are numerous studies that investigate the efficacy of ALBC in reducing the infection rate in primary TKA, there is a paucity of data concerning the possible development of antibiotic resistance resulting from its use. The most important finding of the present study is that there is no difference in the type of periprosthetic knee infection or antibiotic susceptibilities between the ALBC and nonALBC groups, suggesting that the use of ALBC does not increase the risk of antibiotic resistance or affect the pattern of infection.

Prior European hip registry data suggest that the use of ALBC reduces the infection rate among THA. As a result, it has been postulated that this benefit would translate to TKA. ${ }^{19-22)}$ In contrast to these large retrospective studies in the THA population, more recent studies looking at the effect of ALBC on injection rate among TKA do not suggest a benefit to the use of ALBC. In 2009, Namba et al. ${ }^{12)}$ looked at 22,889 patients who underwent TKA with plain bone cement and 2,030 who underwent TKA with ALBC. The infection rate in the ALBC group was 1.4\%, compared to $0.7 \%$ in the plain bone cement group, which was statistically significant $(p=0.002)$. However, it should be noted that the ALBC group had a significantly higher number of comorbidities including diabetes.

Only 2 prospective randomized studies exist in the current literature, which evaluate the efficacy of ALBC in primary TKA. Similar to the literature on ALBC in THA, the results of the prospective randomized studies do not clearly support the findings of the large retrospective studies examining the efficacy of ALBC in reducing infection in TKA. Chiu et al. ${ }^{15}$ performed a randomized study evaluating the efficacy of cefuroxime-impregnated cement in the prevention of deep infection after primary TKAs. Of

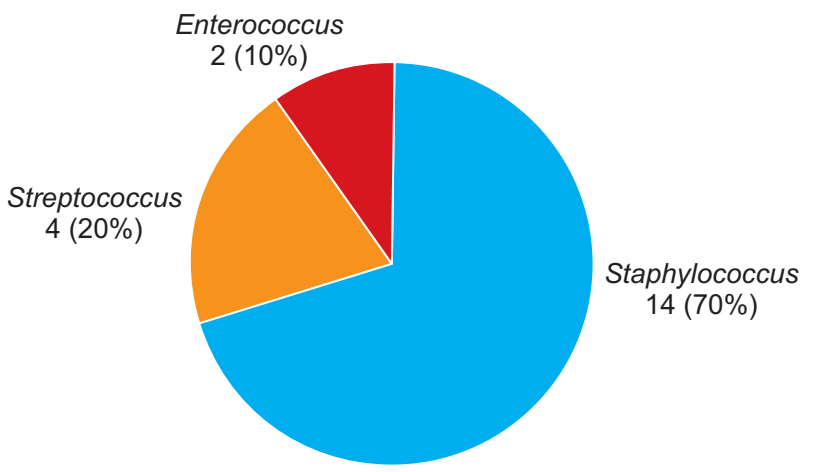

Fig. 2. Infecting organism in total knee arthroplasty utilizing antibioticladen bone cement. 
Schmitt et al. Antibiotic Resistance Due to Antibiotic-Laden Bone Cement

Clinics in Orthopedic Surgery • Vol. 12, No. 3, $2020 \bullet$ www.ecios.org

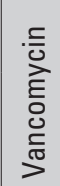 \\ $\sum_{i=1}^{x}$}

es es es es es es as es es

$\sim \sim-\underset{\substack{\mathrm{V} \\ \stackrel{0}{0}}}{\sim} \sim \sim \sim$

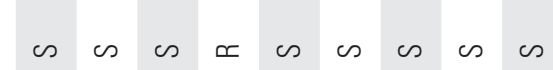

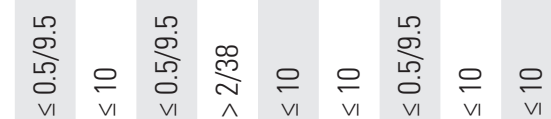

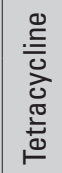

en es es es as as us as es

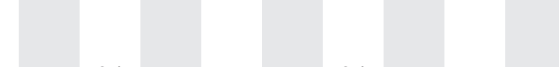

$\begin{array}{lllllllll}\bar{v}_{1} & \sim & \bar{v} & \bar{v} & \bar{v} & \sim & \bar{v} & \bar{v} 1 & \bar{v} 1\end{array}$

ca ca ca ca ca ca ca ca ca

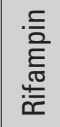

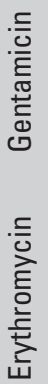

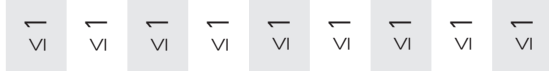

as a cos as a cos as as

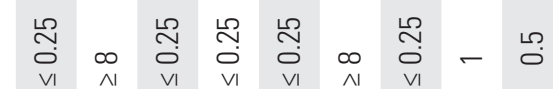

as as as a cos as as es

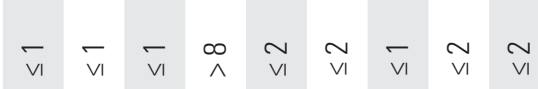

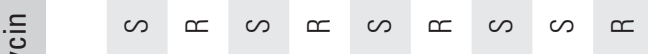

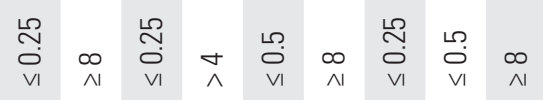

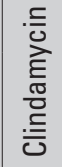

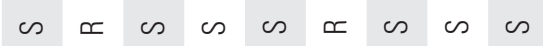

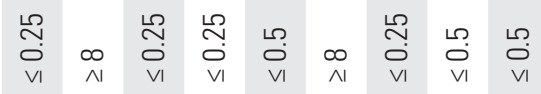

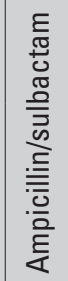

$$
\text { as as as as as as as }
$$

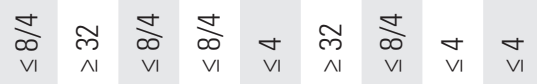

as as as as as as as as as as as as as as $--\sim-----\sim-\cdots-$

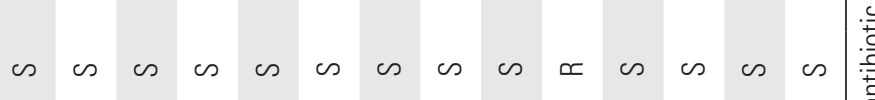

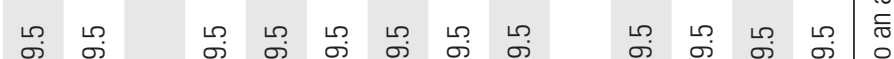

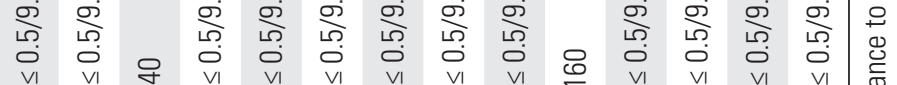

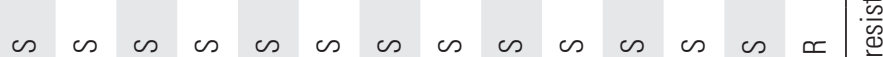

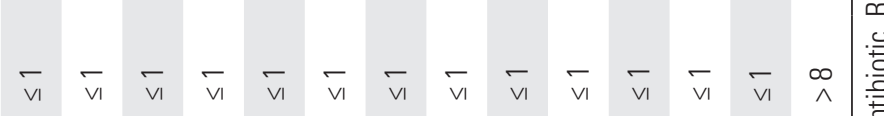

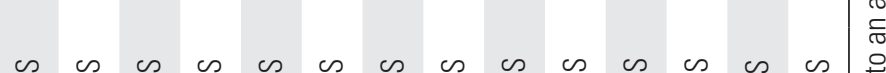

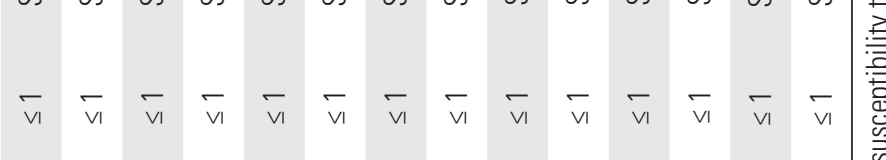

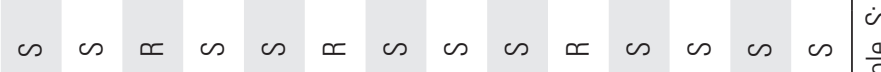

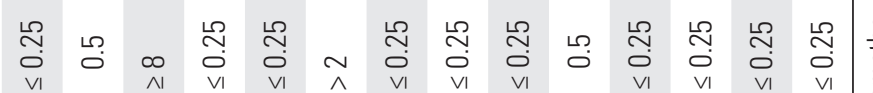

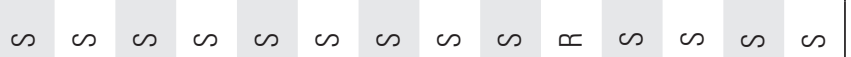

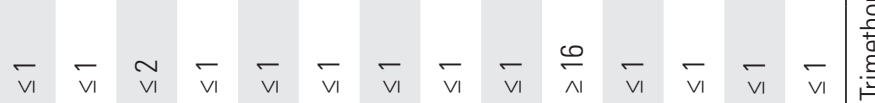

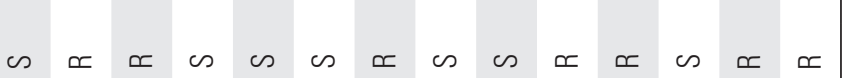

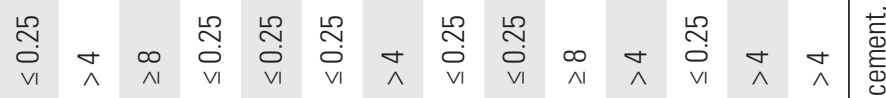

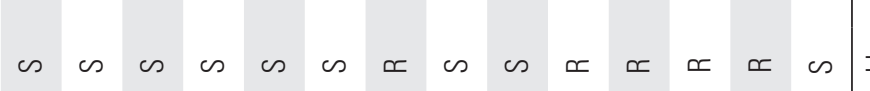

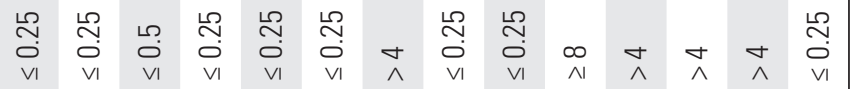

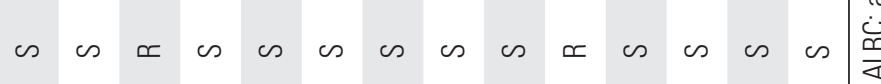

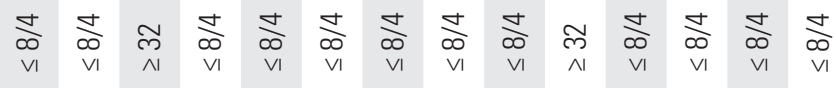


Schmitt et al. Antibiotic Resistance Due to Antibiotic-Laden Bone Cement

Clinics in Orthopedic Surgery • Vol. 12, No. 3, $2020 \bullet$ www.ecios.org

340 TKAs, 178 TKAs were performed with ALBC and 162 with plain bone cement. They reported no infections in the ALBC group and 5 infections in the plain bone cement group, which was statistically significant. Additionally, they concluded that cefuroxime-impregnated cement was effective in the prevention of deep infection in primary TKA in patients with diabetes mellitus based on analysis of a subset of their study population. ${ }^{14)}$ However, this study was performed without clean-air measures such as laminar flow and body-exhaust suits. Subsequently, in 2013, Hinarejos et al. ${ }^{13)}$ reported that the use of erythromycin and colistin-loaded bone cement in TKA did not lead to a decrease in the rate of superficial infection $(1.2 \%$ and $1.8 \%$, respectively; $p=0.53)$ or deep infection $(1.4 \%$ and $1.35 \%$; $p=0.96)$ when systemic prophylactic antibiotics were used.

While there have been only a small number of conflicting studies performed looking at the efficacy of ALBC in reducing infection rates among hip and TKA, even fewer studies have examined the impact of ALBC on antibiotic resistance among patients with hip and knee arthroplasties. Thomes et al. ${ }^{16)}$ used a rat model to compare the rate of infection and profiles of antibiotic resistance in gentamicin-loaded bone cement and those in cement without antibiotic. They found a lower overall infection rate in the gentamicin-loaded cement group, but a significantly higher rate of gentamicin-resistant infection in this group $(p<0.01)$, causing them to conclude gentamicinloaded cement may not be appropriate for revision surgery if it has been used already in previous surgery. Additionally, Sanzen and Walder ${ }^{17)}$ looked at the resistance pattern of CNS from anterior nares swabs of patients, surgeons, operating room staff, and hospital floor staff before and after THA. They reported gentamicin-resistant CNS was only cultured postoperatively in 13 of 79 patients (20\%). Gentamicin-loaded bone cement was used in 10 of 13 of these patients. The only study to look directly at the development of antibiotic resistance among periprosthetic hip infections was by Hope et al. ${ }^{18)}$ in 1989 who found at least 1 strain of gentamicin-resistant CNS in 30 of 34 cases $(88 \%)$ of deep infection in which cement containing gentamicin had been used. In contrast, only 9 of 57 (16\%) in whom antibiotic-free cement was used exhibited gentamicin-resistant CNS. There are no studies, to our knowledge, that examined the impact of ALBC on antibiotic resistance in TKA. Additionally, there are no clinical studies to our knowledge that examined ALBC on antibiotic resistance in bacteria other than CNS.

The current AAOS evidence-based clinical practice guideline for surgical management of osteoarthritis of the knee states that the limited available evidence does not support the routine use of antibiotics in the cement for primary TKA. ${ }^{23)}$ However, its use in the United States continues to increase, particularly in the setting of TKA in high-risk patients, such as those with diabetes or other immunocompromised conditions. ${ }^{4,24)}$ Our study adds further information to the ongoing debate in the arthroplasty literature regarding the use of ALBC in primary TKA. It is the first study to examine the impact of ALBC on antibiotic resistance and infecting organism among TKA, suggesting that the use of ALBC does not confer additional risk in terms of increasing antibiotic resistance or altering the pattern of infecting organism in periprosthetic knee infections.

The limitations of our study include its retrospective nature with the relatively small sample size. Given the low rate of infection among TKA, a larger database study would be needed to support our findings because a prospective randomized study would likely not be feasible. Another potential limitation of this study is that the criteria used by individual surgeons in selecting to use ALBC cannot be accurately determined through review of billing itemization of the surgical admission. Additionally, our single-center study allowed for standardized protocols in terms of infection prevention such as laminar flow, space suits, and preoperative systemic antibiotic prophylaxis, which cannot be controlled for in a larger database study. Further, the efficacy of antibiotic cement was not evaluated, but this was not the aim of this study and this question has been evaluated in multiple prior studies. Finally, while this study examined the impact of antibiotic cement use on antibiotic resistance and infecting organism in periprosthetic knee infections within the same patient, given the focus on antibiotic stewardship to prevent the misuse and overuse of antimicrobials, we were unable to determine the impact of antibiotic cement use on the effects of population-based antibiotic resistance and the spread of multidrug-resistant organisms.

In conclusion, our findings demonstrate that irrespective of the efficacy of ALBC in reducing periprosthetic knee infections, the routine use of ALBC in primary TKA does not increase the risk of antibiotic resistance or pattern of infection. Further research is warranted on this topic as the use of ALBC continues to increase, particularly among high-risk patients.

\section{CONFLICT OF INTEREST}

No potential conflict of interest relevant to this article was reported. 
Schmitt et al. Antibiotic Resistance Due to Antibiotic-Laden Bone Cement

Clinics in Orthopedic Surgery • Vol. 12, No. 3, $2020 \bullet$ www.ecios.org

\section{REFERENCES}

1. Kurtz SM, Ong KL, Lau E, Bozic KJ, Berry D, Parvizi J. Prosthetic joint infection risk after TKA in the Medicare population. Clin Orthop Relat Res. 2010;468(1):52-6.

2. Buchholz HW, Engelbrecht H. Depot effects of various antibiotics mixed with Palacos resins. Chirurg. 1970;41(11):511-5.

3. Chen AF, Parvizi J. Antibiotic-loaded bone cement and periprosthetic joint infection. J Long Term Eff Med Implants. 2014;24(2-3):89-97.

4. Heck D, Rosenberg A, Schink-Ascani M, Garbus S, Kiewitt T. Use of antibiotic-impregnated cement during hip and knee arthroplasty in the United States. J Arthroplasty. 1995;10(4):470-5.

5. Espehaug B, Engesaeter LB, Vollset SE, Havelin LI, Langeland N. Antibiotic prophylaxis in total hip arthroplasty: review of 10,905 primary cemented total hip replacements reported to the Norwegian arthroplasty register, 1987 to 1995. J Bone Joint Surg Br. 1997;79(4):590-5.

6. Dale H, Hallan G, Hallan G, Espehaug B, Havelin LI, Engesaeter LB. Increasing risk of revision due to deep infection after hip arthroplasty. Acta Orthop. 2009;80(6):639-45.

7. Engesaeter LB, Espehaug B, Lie SA, Furnes O, Havelin LI. Does cement increase the risk of infection in primary total hip arthroplasty? Revision rates in 56,275 cemented and uncemented primary THAs followed for 0-16 years in the Norwegian Arthroplasty Register. Acta Orthop. 2006;77(3):351-8.

8. Zhou Y, Li L, Zhou Q, et al. Lack of efficacy of prophylactic application of antibiotic-loaded bone cement for prevention of infection in primary total knee arthroplasty: results of a meta-analysis. Surg Infect (Larchmt). 2015;16(2):183-7.

9. Wang H, Qiu GX, Lin J, Jin J, Qian WW, Weng XS. Antibiotic bone cement cannot reduce deep infection after primary total knee arthroplasty. Orthopedics. 2015;38(6):e462-6.

10. Wang J, Zhu C, Cheng T, et al. A systematic review and meta-analysis of antibiotic-impregnated bone cement use in primary total hip or knee arthroplasty. PLoS One. 2013;8(12):e82745.

11. Schiavone Panni A, Corona K, Giulianelli M, Mazzitelli G, Del Regno C, Vasso M. Antibiotic-loaded bone cement reduces risk of infections in primary total knee arthroplasty? A systematic review. Knee Surg Sports Traumatol Arthrosc. 2016;24(10):3168-74.

12. Namba RS, Chen Y, Paxton EW, Slipchenko T, Fithian DC. Outcomes of routine use of antibiotic-loaded cement in primary total knee arthroplasty. J Arthroplasty. 2009;24(6 Suppl):44-7.

13. Hinarejos P, Guirro P, Leal J, et al. The use of erythromy- cin and colistin-loaded cement in total knee arthroplasty does not reduce the incidence of infection: a prospective randomized study in 3000 knees. J Bone Joint Surg Am. 2013;95(9):769-74.

14. Chiu FY, Lin CF, Chen CM, Lo WH, Chaung TY. Cefuroxime-impregnated cement at primary total knee arthroplasty in diabetes mellitus: a prospective, randomized study. J Bone Joint Surg Br. 2001;83(5):691-5.

15. Chiu FY, Chen CM, Lin CF, Lo WH. Cefuroxime-impregnated cement in primary total knee arthroplasty: a prospective, randomized study of three hundred and forty knees. J Bone Joint Surg Am. 2002;84(5):759-62.

16. Thomes B, Murray P, Bouchier-Hayes D. Development of resistant strains of Staphylococcus epidermidis on gentamicin-loaded bone cement in vivo. J Bone Joint Surg Br. 2002;84(5):758-60.

17. Sanzen L, Walder M. Antibiotic resistance of coagulase-negative staphylococci in an orthopaedic department. J Hosp Infect. 1988;12(2):103-8.

18. Hope PG, Kristinsson KG, Norman P, Elson RA. Deep infection of cemented total hip arthroplasties caused by coagulase-negative staphylococci. J Bone Joint Surg Br. 1989;71(5):851-5.

19. Engesaeter LB, Lie SA, Espehaug B, Furnes O, Vollset SE, Havelin LI. Antibiotic prophylaxis in total hip arthroplasty: effects of antibiotic prophylaxis systemically and in bone cement on the revision rate of 22,170 primary hip replacements followed 0-14 years in the Norwegian Arthroplasty Register. Acta Orthop Scand. 2003;74(6):644-51.

20. Malchau H, Herberts P, Ahnfelt L. Prognosis of total hip replacement in Sweden: follow-up of 92,675 operations performed 1978-1990. Acta Orthop Scand. 1993;64(5):497-506.

21. McQueen M, Littlejohn A, Hughes SP. A comparison of systemic cefuroxime and cefuroxime loaded bone cement in the prevention of early infection after total joint replacement. Int Orthop. 1987;11(3):241-3.

22. Josefsson G, Kolmert L. Prophylaxis with systematic antibiotics versus gentamicin bone cement in total hip arthroplasty: a ten-year survey of 1,688 hips. Clin Orthop Relat Res. 1993;(292):210-4.

23. Weber KL, Jevsevar DS, McGrory BJ. AAOS clinical practice guideline: surgical management of osteoarthritis of the knee: evidence-based guideline. J Am Acad Orthop Surg. 2016;24(8):e94-6.

24. Bourne RB. Prophylactic use of antibiotic bone cement: an emerging standard-in the affirmative. J Arthroplasty. 2004;19 (4 Suppl 1):69-72. 